\title{
In-medium modified energy-momentum tensor form factors
}

\author{
Ju-Hyun Jung*, Ulugbek Yakhshiev ${ }^{\dagger}$ and Hyun-Chul Kim ${ }^{\ddagger}$ \\ Department of Physics, Inha University, 402-751 \\ Incheon, Republic of Korea \\ *juhyun@inha.edu \\ †yakhshiev@inha.ac.kr \\ ¥hckim@inha.ac.kr
}

Published 16 April 2014

\begin{abstract}
In this talk, we report a recent investigation on the energy-momentum tensor form factors of the nucleon in nuclear medium, based on the framework of the in-medium modified chiral soliton model. The model was constructed by taking into account the influence of the surrounding environment to the mesonic sector $(\pi-, \rho$ - and $\omega$-meson properties). We briefly discuss the results of the energy-momentum tensor form factors.
\end{abstract}

Keywords: Skyrmions; protons and neutrons; mesons in nuclear matter.

PACS Numbers: 12.39 Dc, 14.20.Dh, 21.65.Jk

\section{Introduction}

It is of great importance to study the energy-momentum tensor form factors (EMTFFs), since they shed light on the structure of the nucleon from a new direction. They also provide certain information on how the constituents of the nucleon behave inside a nucleon. The EMTFFs have become interesting issue only very recently. The reason is that the probe of measuring them is the graviton, so that it is almost impossible to get access experimentally to them. However, the generalized parton distributions (GPDs) makes it possible to investigate the EMTFFs from hard exclusive reactions, ${ }^{1-4}$ since the Melin transforms of certain GPDs are connected to the EMTFFs that explain how the mass and the spin are distributed inside a nucleon.

The nucleon matrix element of the totally symmetric energy-momentum tensor (EMT) operator can be parameterized in terms of three real form factors defined as follows: ${ }^{5,6}$

This is an Open Access article published by World Scientific Publishing Company. It is distributed under the terms of the Creative Commons Attribution 3.0 (CC-BY) License. Further distribution of this work is permitted, provided the original work is properly cited. 


$$
\begin{aligned}
\left\langle p^{\prime}\left|\hat{T}_{\mu \nu}(0)\right| p\right\rangle= & \bar{u}\left(p^{\prime}, s^{\prime}\right)\left[M_{2}(t) \frac{P_{\mu} P_{\nu}}{M_{N}}+J(t) \frac{i\left(P_{\mu} \sigma_{\nu \rho}+P_{\nu} \sigma_{\mu \rho}\right) \Delta^{\rho}}{2 M_{N}}\right. \\
& \left.+d_{1}(t) \frac{\Delta_{\mu} \Delta_{\nu}-g_{\mu \nu} \Delta^{2}}{5 M_{N}}\right] u(p, s),
\end{aligned}
$$

where $P=\left(p+p^{\prime}\right) / 2, \Delta=\left(p^{\prime}-p\right)$ and $t=\Delta^{2}$. The $M_{N}$ and $u(p, s)$ denote the nucleon mass and spinor, respectively. The form factor $M_{2}(t)$ gives information about the ratio of the momenta carried by constituents of a nucleon. In QCD, $M_{2}(t)$ at the zero-momentum transfer shows that about a half of the momentum of a fast moving nucleon is carried by quarks, and the other half by gluons. The other form factor $J(t)$ is related to the total angular momentum of the quark and gluons, though it is not much known experimentally. It is also important to understand the last form factor $d_{1}(t)$ in Eq. (1), since it describes how the strong forces are distributed and stabilized in the nucleon. ${ }^{6,7}$ It can be extracted from the beam charge asymmetry in deeply virtual Compton scattering. ${ }^{8}$

The EMTFFs, in particular, the pressure plays a role of a touchstone for any model. It contrains the model in such a way that the pressure should be exactly equal to zero. Moreover, the $D$-term is in deep connection to the spontaneous breakdown of chiral symmetry. ${ }^{8-10}$ Thus, the EMTFFs pave the way to understand the srtucture of the nucleon with a whole new perspective.

Recently, we studied EMTFFs of the free nucleon in framework of the chiral solitonic model which includes the explicit $\pi, \rho$ and $\omega$ mesonic degrees of freedom. ${ }^{11}$ The results of the EMTFFs showed how the $\pi$ and vector mesons shape the nucleon as a soliton. In particular, the pion tail of the pressure distribution explicitly showed that the outer part of the nucleon is governed only by the pionic degrees of freedom, while the core part is described by the vector mesons. In this context, it is of great interest to extend this recent investigation ${ }^{11}$ to nuclear matter based on a mediummodified model. ${ }^{12}$ The results in detail will appear elsewhere. ${ }^{13}$

\section{Lagrangian of the Model}

We start with the in-medium modified effective chiral Lagrangian with the $\pi, \rho$, and $\omega$ meson degrees of freedom, where the nucleon arises as a topological solution. The Lagrangian is written as ${ }^{\text {a }}$

$$
\begin{aligned}
\mathcal{L}^{*}= & \frac{f_{\pi}^{2}}{4} \operatorname{Tr}\left(\partial_{0} U \partial_{0} U^{\dagger}\right)-\alpha_{p} \frac{f_{\pi}^{2}}{4} \operatorname{Tr}\left(\partial_{i} U \partial_{i} U^{\dagger}\right)+\alpha_{s} \frac{f_{\pi}^{2} m_{\pi}^{2}}{2} \operatorname{Tr}(U-1) \\
& +\alpha_{s} \frac{f_{\pi}^{2} m_{\pi}^{2}}{2} \operatorname{Tr}(U-1)+\frac{a}{4} f_{\pi}^{2} \operatorname{Tr}\left[D_{\mu} \xi \cdot \xi^{\dagger}+D_{\mu} \xi^{\dagger} \cdot \xi\right]^{2}-\frac{1}{2 g^{2} \zeta} \operatorname{Tr}\left(F_{\mu \nu}^{2}\right)
\end{aligned}
$$

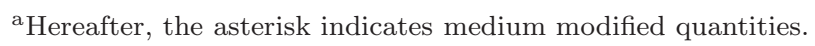




$$
+\left(\frac{N_{c}}{2} g \sqrt{\zeta}\right) \omega_{\mu} \frac{\epsilon^{\mu \nu \alpha \beta}}{24 \pi^{2}} \operatorname{Tr}\left\{\left(U^{\dagger} \partial_{\nu} U\right)\left(U^{\dagger} \partial_{\alpha} U\right)\left(U^{\dagger} \partial_{\beta} U\right)\right\},
$$

where $U=\xi_{L}^{\dagger} \xi_{R}$, and the field-strength tensor and the covariant derivative are defined, respectively, as

$$
\begin{aligned}
F_{\mu \nu} & =\partial_{\mu} V_{\nu}-\partial_{\nu} V_{\mu}-i\left[V_{\mu}, V_{\nu}\right], \\
D_{\mu} \xi_{L(R)} & =\partial_{\mu} \xi_{L(R)}-i V_{\mu} \xi_{L(R)} .
\end{aligned}
$$

The pion decay constant $f_{\pi}$ and the pion mass $m_{\pi}$ are fixed by experimental data: $f_{\pi}=93 \mathrm{MeV}$ and $m_{\pi}=135 \mathrm{MeV}$ (the neutral pion mass). The number of colors is taken to be $N_{c}=3$ and the coupling constant $g$ is related to the KSRF relation $m_{\rho}^{2}=m_{\omega}^{2}=a g^{2} \zeta f_{\pi}^{2}$ with $a=2$ such that we have $\rho \pi \pi$ coupling in free space $g_{\rho \pi \pi}=a g / 2$ and $g=5.85$. Note that the $g_{\rho \pi \pi}$ is close to its empirical value $g_{\rho \pi \pi}=$ 6.11.

The medium functionals defined as

$$
\alpha_{p}=1-\frac{4 \pi c_{0} \rho / \eta}{\left.1+g_{0}^{\prime} 4 \pi c_{0} \rho\right) / \eta}, \quad \alpha_{s}=1-\frac{4 \pi \eta b_{0} \rho}{m_{\pi}^{2}}, \quad \zeta=\exp \left\{-\frac{\gamma_{\mathrm{num}} \rho}{1+\gamma_{\operatorname{den}} \rho}\right\} .
$$

depend on the nuclear matter density $\rho$ and represent the influence of surrounding nuclear environment to single soliton properties. ${ }^{12}$ In the expressions presented in Eq. (5) $\eta=1+m_{\pi} / m_{N} \sim 1.14$ is a kinematical factor. The values of the empirical parameters $b_{0}=-0.024 m_{\pi}^{-1}$ and $c_{0}=0.09 m_{\pi}^{-3}$ are taken from the analyses of pionic atoms and low-energy pion-nucleus scattering data. The $g_{0}^{\prime}=0.7$ denotes the Lorentz-Lorenz factor that takes into account the short-range correlations. ${ }^{14}$ The values of $\gamma_{\text {num }}=2.390 m_{\pi}^{-3}$ and $\gamma_{\text {den }}=1.172 m_{\pi}^{-3}$ are chosen in a such way that the properties of the nuclear matter around the normal nuclear matter density is reproduced in a correct way.

Assuming the following Ansätze for the pseudoscalar and vector mesons

$$
\begin{aligned}
& U=\exp \left\{\frac{i \boldsymbol{\tau} \cdot \boldsymbol{r}}{r} F(r)\right\}, \quad V_{\mu}=\frac{g}{2}\left(\boldsymbol{\tau} \cdot \boldsymbol{\rho}_{\mu}+\omega_{\mu}\right), \\
& \rho_{0}^{a}=0, \quad \rho_{i}^{a}=\frac{\varepsilon_{i k a} r_{k}}{g \sqrt{\zeta} r^{2}} G(r), \quad \omega_{\mu}=\omega(r) \delta_{\mu 0}
\end{aligned}
$$

one can minimize the static mass functional related to the Lagrangian (2) and find the solitonic solutions correponding to the unit baryon number $(B=1)$. The integrand of the mass static mass functional corresponds to $T^{00}$ component of the energy momentum tensor presented below. The details of the minimization procedure can be found in Ref. 12 .

Using the Lagrangian in Eq. (2), one can calculate each component of the EMT as follows:

$$
\begin{aligned}
T^{00}(r)= & \alpha_{p} \frac{f_{\pi}^{2}}{2}\left(2 \frac{\sin ^{2} F}{r^{2}}+F^{\prime 2}\right)+\alpha_{s} f_{\pi}^{2} m_{\pi}^{2}(1-\cos F) \\
& +\frac{2 f_{\pi}^{2}}{r^{2}}(1-\cos F+G)^{2}-\zeta g^{2} f_{\pi}^{2} \omega^{2}
\end{aligned}
$$




$$
\begin{gathered}
+\frac{1}{2 g^{2} \zeta r^{2}}\left\{2 r^{2} G^{2}+G^{2}(G+2)^{2}\right\}-\frac{1}{2} \omega^{\prime 2} \\
+\left(\frac{3}{2} g \sqrt{\zeta}\right) \frac{1}{2 \pi^{2} r^{2}} \omega \sin ^{2} F F^{\prime}, \\
T^{0 i}(\boldsymbol{r}, \boldsymbol{s})=\frac{e^{i l m} r^{l} s^{m}}{(\boldsymbol{s} \times \boldsymbol{r})^{2}} \rho_{J}(r), \\
T^{i j}(r)=s(r)\left(\frac{r^{i} r^{j}}{r^{2}}-\frac{1}{3} \delta^{i j}\right)+p(r) \delta^{i j},
\end{gathered}
$$

where

$$
\begin{aligned}
\rho_{J}(r)= & \frac{f_{\pi}^{2}}{3 \lambda}\left[\sin ^{2} F+8 \sin ^{4} \frac{F}{2}-4 \sin ^{2} \frac{F}{2} \xi_{1}\right] \\
& +\frac{1}{3 g^{2} r^{2} \zeta \lambda}\left[\left(2-2 \xi_{1}-\xi_{2}\right) G^{2}\right] \\
& +\frac{g \sqrt{\zeta}}{8 \pi^{2} \lambda} \Phi \sin ^{2} F F^{\prime}
\end{aligned}
$$

is the angular momentum density and

$$
\begin{aligned}
p(r)= & -\frac{1}{6} \alpha_{p} f_{\pi}^{2}\left(F^{\prime 2}+2 \frac{\sin ^{2} F}{r^{2}}\right)-\alpha_{s} f_{\pi}^{2} m_{\pi}^{2}(1-\cos F) \\
& -\frac{2}{3 r^{2}} f_{\pi}^{2}(1-\cos F+G)^{2}+f_{\pi}^{2} g^{2} \zeta \omega^{2} \\
& +\frac{1}{6 g^{2} \zeta r^{2}}\left\{2 r^{2} G^{\prime 2}+G^{2}(G+2)^{2}\right\}+\frac{1}{6} \omega^{\prime 2}, \\
s(r)= & \alpha_{p} f_{\pi}^{2}\left(F^{\prime 2}-\frac{\sin ^{2} F}{r^{2}}\right)-\frac{2 f_{\pi}^{2}}{r^{2}}(1-\cos F+G)^{2} \\
& +\frac{1}{g^{2} r^{2} \zeta}\left\{r^{2} G^{2}-G^{2}(G+2)^{2}\right\}-\omega^{\prime 2}
\end{aligned}
$$

are the pressure and shear force distributions inside the nucleon. The moment of inertia of the rotating soliton including $1 / N_{c}$ corrections is given by the expression

$$
\begin{aligned}
\lambda=4 \pi \int d r & {\left[\frac{2 f_{\pi}^{2}}{3}\left(\sin ^{2} F+8 \sin ^{4} \frac{F}{2}-4 \sin ^{2} \frac{F}{2} \xi_{1}\right)\right.} \\
& \left.+\frac{2}{3 g^{2} r^{2} \zeta}\left\{\left(2-2 \xi_{1}-\xi_{2}\right) G^{2}\right\}+\frac{g \sqrt{\zeta}}{4 \pi^{2}} \Phi \sin ^{2} F F^{\prime}\right] .
\end{aligned}
$$

As we mentioned above, the integral of $T_{00}$ gives the soliton mass at the zero momentum transfer $t=0$. Therefore, The $M_{2}(t)$ form factor is normalized by the nucleon 
mass as

$$
M_{2}(0)=\frac{1}{M_{N}} \int_{0}^{\infty} \mathrm{d}^{3} \mathrm{r} T_{00}(r)=1
$$

in the leading order $M_{N}$, which is equals to the soliton mass.

\section{Results}

The results of calculations are listed in Table 1 for free space case and in nuclear matter at normal nuclear matter density $\rho=\rho_{0}$. For comparison we present some results corresponding to the in-medium modified Skyrme model for both the free nucleons and for the nucleons at normal nuclear matter density ${ }^{15}$ and chiral quarksoliton model ${ }^{17}$ for the nucleons in free space.

One can see that the mean square radii of the energy distribution are more or less similar in all models but the mean square radii of the angular distribution are

Table 1. The quantities relevant to the nucleon EMT densities and their form factors: $\left\langle r_{00}^{2}\right\rangle$ are the mean square radii for the energy densities; $\left\langle r_{J}^{2}\right\rangle$ represent the squared radii of the angular momentum distribution; $r_{0}$ designates the position, where the sign of the pressure is changed; $d_{1}(0)$ correspond to the $d_{1}(t)$ form factors at the zero momentum transfer; $p_{0}(0)$ denote the pressure values at the origin. Each quantities are represented at free space $\rho=0$ and at the normal nuclear matter density $\rho=\rho_{0}$.

\begin{tabular}{ccccccccccc}
\hline & $\begin{array}{c}f_{\pi} \\
{[\mathrm{MeV}]}\end{array}$ & $\begin{array}{c}\left\langle r_{E}^{2}\right\rangle \\
{\left[\mathrm{fm}^{2}\right]}\end{array}$ & $\begin{array}{c}\left\langle r_{J}^{2}\right\rangle \\
{\left[\mathrm{fm}^{2}\right]}\end{array}$ & \multicolumn{2}{c}{$\begin{array}{c}r_{0} \\
{[\mathrm{fm}]}\end{array}$} & & \multicolumn{2}{c}{$d_{1}(0)$} \\
\hline$\rho / \rho_{0}$ & & 0 & 1 & 0 & 1 & 0 & 1 & 0 & 1 \\
\hline$\pi-\rho-\omega$ mesonic model $_{\text {Skyrme model }}^{15}$ & 93 & 0.78 & 1.06 & 0.74 & 0.77 & 0.55 & 0.65 & -5.03 & -5.59 \\
Chiral quark solton model $^{17}$ & 54 & 0.68 & 0.95 & 1.09 & 1.35 & 0.71 & 0.90 & -3.54 & -4.85 \\
\hline
\end{tabular}

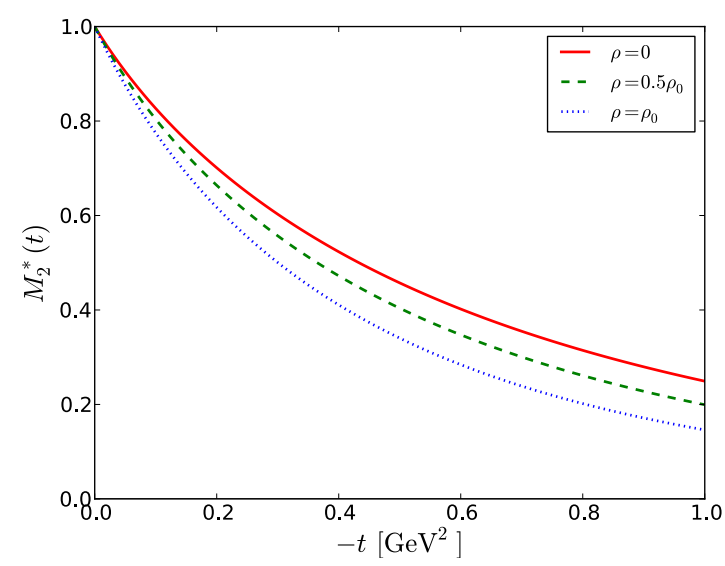

Fig. 1. $\quad M_{2}^{*}(t)$ as a function of $t=-\Delta^{2}$ for the nucleon with the nuclear matter density varied as shown in the legend. 


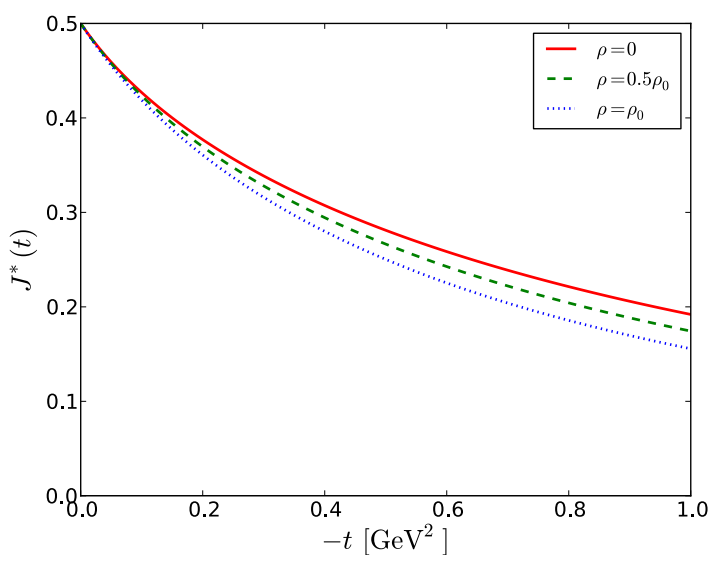

Fig. 2. $J^{*}(t)$ as a function of $t=-\Delta^{2}$ for the nucleon with the nuclear matter density varied as shown in the legend.

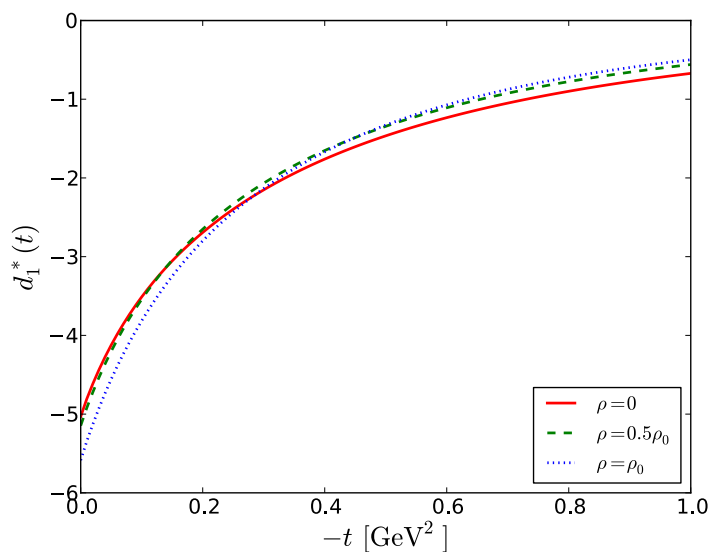

Fig. 3. $d_{1}^{*}(t)$ as a function of $t=-\Delta^{2}$ for the nucleon with the nuclear matter density varied as shown in the legend.

distinguished. The point at which the pressure is changed is similar in the case of the $\pi-\rho-\omega$ model.

In Figures 1, 2 and 3 we show the momentum dependence of the EMTFFs.

One can see that with the increasing the momentum transfer form factors in the nuclear medium fall off faster than those in free space.

\section{Summary}

In summary, we have considered the energy-momentum tensor form factors in the framework of the in-medium modified $\pi-\rho-\omega$ model. The results are in qualitative 
agreement with other model calculations in free space ${ }^{15,17}$ as well as in nuclear matter. $^{15}$

\section{Acknowledgments}

This work is supported by the Basic Science Research Program through the National Research Foundation (NRF) of Korea funded by the Korean government (Ministry of Education, Science and Technology), Grant No. 2012-0008469 (J.H.J. and U.Y.) and Grant No. 2012004024 (H.Ch.K.).

\section{References}

1. D. Müller, D. Robaschik, B. Geyer, F. M. Dittes and J. Hor̆ejs̆i, Fortsch. Phys. 42 (1994) 101.

2. X. D. Ji, Phys. Rev. D 55 (1997) 7114.

3. J. C. Collins, L. Frankfurt and M. Strikman, Phys. Rev. D 56 (1997) 2982.

4. A. V. Radyushkin, Phys. Rev. D 56 (1997) 5524.

5. X. D. Ji, Phys. Rev. Lett. 78 (1997) 610.

6. M. V. Polyakov, Phys. Lett. B 555 (2003) 57.

7. M. V. Polyakov and A. G. Shuvaev, hep-ph/0207153.

8. N. Kivel, M. V. Polyakov and M. Vanderhaeghen, Phys. Rev. D 63 (2001) 114014.

9. M. V. Polyakov and C. Weiss, Phys. Rev. D 60 (1999) 114017.

10. K. Goeke, M. V. Polyakov and M. Vanderhaeghen, Prog. Part. Nucl. Phys. 47 (2001) 401.

11. J.-H. Jung, U. Yakhshiev and H.-C. Kim, arXiv:1310.8064 [hep-ph].

12. J.-H. Jung, U. T. Yakhshiev and H.-Ch. Kim, Phys. Lett. B 723, 442 (2013).

13. J.-H. Jung, U. T. Yakhshiev and H.-Ch. Kim, in preparation.

14. T. Ericson and W. Weise, Pions and Nuclei (Clarendon, Oxford, 1988).

15. H.-Ch. Kim, P. Schweitzer and U. Yakhshiev, Phys. Lett. B 718, 625 (2012).

16. C. Cebulla, K. Goeke, J. Ossmann and P. Schweitzer, Nucl. Phys. A 794, 87 (2007).

17. K. Goeke et al., Phys. Rev. D 75, 094021 (2007). 